\title{
Estimulación musical para las funciones cognitivas del inglés en la educación superior
}

\section{Musical stimulation for cognitive functions of English in higher education}

Silvana Patricia Célleri Quinde. ${ }^{1}$, Mónica Alejandra Logroño Becerra. ${ }^{2}$, Edison Renato Ruiz López. ${ }^{3} \&$ Edwin Alberto Inca Chunata. ${ }^{4}$

Recibido: 11-02-2020 / Revisado: 18-03-2020 /Aceptado: 04-04-2020/ Publicado: 08-05-2020

\begin{abstract}
.
DOI: https://doi.org/10.33262/concienciadigital.v3i2.1.1233

The objective of this work is to show the results of an experiment carried out with groups of students of different ages to whom musicology was apply as a method of learning the English language. By stimulating the auditory sense in the different test groups, the skills assimilated by students from three different environments in their training and characteristics were identified, the first a group of thirty students from the Faculty of Business Administration, the second, a group of 30 students from the Faculty of Sciences and the third group of 40 high school students. The experiment concludes that the method is efficient and achieves a significant improvement in the learning of the English language; the hypothesis was test applying the Student's t-statistic for correlated groups in which the previous performance was compare with the postmethod performance in a group of 10 randomly selected students.
\end{abstract}

Keywords: Musicology, English Language, Method, Learning, Skill

\section{Resumen.}

Este trabajo tiene por objeto mostrar los resultados de un experimento planteado con grupos de estudiantes de diferentes edades a quienes se les aplicó musicología como un método de aprendizaje del idioma inglés. Al estimular el sentido auditivo en los

\footnotetext{
${ }^{1}$ Escuela Superior Politécnica de Chimborazo, Facultad de Administración de Empresas. Riobamba, Ecuador. patricia.celleri@espoch.edu.ec

${ }^{2}$ Escuela Superior Politécnica de Chimborazo, Facultad de Administración de Empresas. Riobamba, Ecuador. malogroño@espoch.edu.ec

${ }^{3}$ Escuela Superior Politécnica de Chimborazo, Facultad de Ciencias. Riobamba, Ecuador. edison.ruiz@espoch.edu.ec

${ }^{4}$ Ministerio de Educación, Educación Cultural y Artística. Sangolquí, Ecuador. edwin.inca@educacion.gob.ec
} 
diferentes grupos de prueba se identificaron las destrezas asimiladas por los estudiantes de tres entornos distintos en su formación y características, el primero un grupo de treinta estudiantes de la Facultad de Administración de Empresas, el segundo, un grupo de 30 estudiantes de la Facultad de Ciencias y el tercer grupo de 40 estudiantes de educación secundaria. El experimento concluye que el método es eficiente y logra una mejora significativa en el aprendizaje del idioma inglés, la hipótesis fue probada aplicando el estadístico T de Student para grupos correlacionados en el que se comparó el rendimiento anterior con el posterior al método en un grupo de 10 estudiantes seleccionados de manera aleatoria.

Palabras claves: Musicología, Lenguaje Inglés, Método, Aprendizaje, Destreza

\section{Introducción.}

Los métodos juegan un papel central en la enseñanza del idioma inglés. Las clases modernas de inglés se caracterizan por una variedad de métodos. Un método es el proceso (el "cómo") de impartir conocimiento, es decir, la forma profesional y orientada al alumno de aprender objetivos y contenido de aprendizaje. Los métodos de enseñanza del inglés difieren entre los métodos tradicionales y los métodos alternativos. Los métodos tradicionales son aquellos que han sido 'probados' en las clases de inglés y todavía se usan en la clase de hoy. Como regla, los métodos alternativos no han podido establecerse. (Beltrán, 2017)

\section{Métodos Tradicionales}

El método de traducción gramatical. Este método se basa en la enseñanza de idiomas tradicionales (especialmente el latín), aparece desde el siglo XIX hasta mediados del siglo XX. Se caracteriza por la traducción de ida y vuelta de oracione individuales, a menudo no relacionadas. El objetivo más importante de las lecciones es el conocimiento del idioma y no las habilidades del idioma. La pronunciación es descuidada. Las reglas gramaticales deben memorizarse y usarse en la construcción de oraciones como parte de la traducción. (GarcíaMedall Villanueva, 2001)

El método directo. El método directo es utilizado por Wilhelm y otros representantes del movimiento de reforma ( (Darian, 1969) éste método es requerido para la enseñanza de lenguas extranjeras desde 1880. Rechazan el método de traducción gramatical y abogan por lecciones inductivas de gramática (es decir, "de los ejemplos a la regla"). Otro requisito de los reformadores es la introducción de material de lectura escolar, ya que ya no debería ser un tema aislado de aprendizaje en el aula (como con el método de traducción gramatical), sino textos completos. Las habilidades lingüísticas son más importantes que el conocimiento del idioma (énfasis en la pronunciación y el habla fluida; transcripción fonética). (Beltrán, 2017) 
El método audiolinguie. Las habilidades lingüísticas son más importantes que el conocimiento del idioma (énfasis en la pronunciación y el habla fluida; transcripción fonética). Por lo tanto, las lecciones deben ser consistentemente en el idioma extranjero (monolingüe). (Bernal Vázquez, 2010)

La musicología ha dado forma a la enseñanza del inglés desde la década de 1950 hasta la de 1970. Fue desarrollado en los Estados Unidos en la década de 1940 y se utilizó para preparar a los soldados estadounidenses para operaciones en el extranjero (Programa de Entrenamiento Especializado del Ejército, "método del ejército"). La musicología se basa en los resultados del aprendizaje y la psicología del comportamiento (conductismo) y la lingüística (estructuralismo). El punto de partida de este método es la suposición de que hablar es actuar y, por lo tanto, puede ser influenciado. En el llamado "procedimiento mimmem" ( mimetización-memorización), los patrones lingüísticos se deben "enlazar" mediante una imitación constante (Castro Martínez, 2014)

Mediante la musicología se desarrollan hábitos de habla automatizados. Las oraciones modelo deben practicarse hasta el sobre aprendizaje. Las clases se imparten en el idioma extranjero y la atención se centra en la producción del lenguaje oral. El medio más importante del método de audio lingual es el laboratorio de idiomas, que asegura el más alto grado de individualización y objetivación del proceso de aprendizaje. (Castro Martínez, 2014). En lugar del aprendizaje consciente, la práctica intensiva (sin la participación del control de la conciencia) se lleva a cabo de acuerdo con el principio de estímulo-respuesta, por ejemplo:

El método bilingüe El método bilingüe fue desarrollado por CJ Dodson en la década de 1960 y propagado en la República Federal por W. Butzkamm ("monolingüismo ilustrado") en la década de 1970. Es un aprendizaje basado en la percepción. Un desarrollo muy nuevo es la enseñanza de asignaturas bilingües, en la cual las asignaturas como historia o geografía se imparten en inglés. (Pérez Morales, 2008)

El enfoque comunicativo. En el curso del llamado giro comunicativo a principios de la década de 1970, hay un desarrollo de largo alcance en la enseñanza de lenguas extranjeras, que ha sido de gran importancia hasta el presente. El alumno debe estar capacitado para lidiar con situaciones de idiomas extranjeros de manera comunicativa, es decir, debe poder utilizar los medios de habla apropiados y relevantes.

Superlearning Con este enfoque, el alumno asume una nueva identidad y, a menudo, se embarca en un viaje imaginario, tienen experiencia en situaciones cotidianas. Un principio de este método es el desmantelamiento de las barreras de aprendizaje (miedo, estrés, etc.) a través de la relajación profunda (música) y una atmósfera armoniosa y estimulante. El alumno debe encontrarse a sí mismo (centrado). El proceso de aprendizaje tiene lugar en gran medida en el subconsciente. (Bustos Flores, 2012) 
El camino silencioso Este enfoque fue desarrollado por Caleb Gattegno. El profesor no hace correcciones explícitas, en su mayoría calla y acompaña el proceso de aprendizaje de sus alumnos. La fonética es de importancia central. El lenguaje primero se divide en sus componentes más pequeños y luego se vuelve a ensamblar (síntesis). (Pérez Morales, 2008)

Aprendizaje del lenguaje comunitario La responsabilidad de los temas y contenidos de la conversación recae en los alumnos, quienes desarrollan preguntas sobre el idioma extranjero junto con el profesor y buscan respuestas. El profesor es consultor y compañero. El aprendizaje de lenguas extranjeras se lleva a cabo a través de la interacción de los miembros de un grupo de aprendizaje. (Darian, 1969)

El enfoque natural Este método fue desarrollado por Tracy Terrell y Stephen Krashen en la década de 1970. Asumen que el idioma extranjero se adquiere como la lengua materna (proceso inconsciente) y no se debe aprender (proceso consciente). El alumno solo comienza a hablar cuando se siente listo. Con el Enfoque natural, el enfoque está en el lenguaje oral. (Escobar, 2005)

Se puede describir el proceso de aprendizaje de un idioma utilizando la ilustración presentada en la Fig. 1. La adquisición de una habilidad por parte del alumno se muestra, en este caso, conocimiento del idioma inglés En el primer año, el estudiante adquiere el 25-30\% de las habilidades de idiomas extranjeros, expandiendo esta habilidad en el segundo año, a través de discusiones y prácticas, en el tercer año al transferir la habilidad a otros estudiantes.

Figura 1. Pirámide de los niveles de conocimiento

\section{Learning Pyramid}

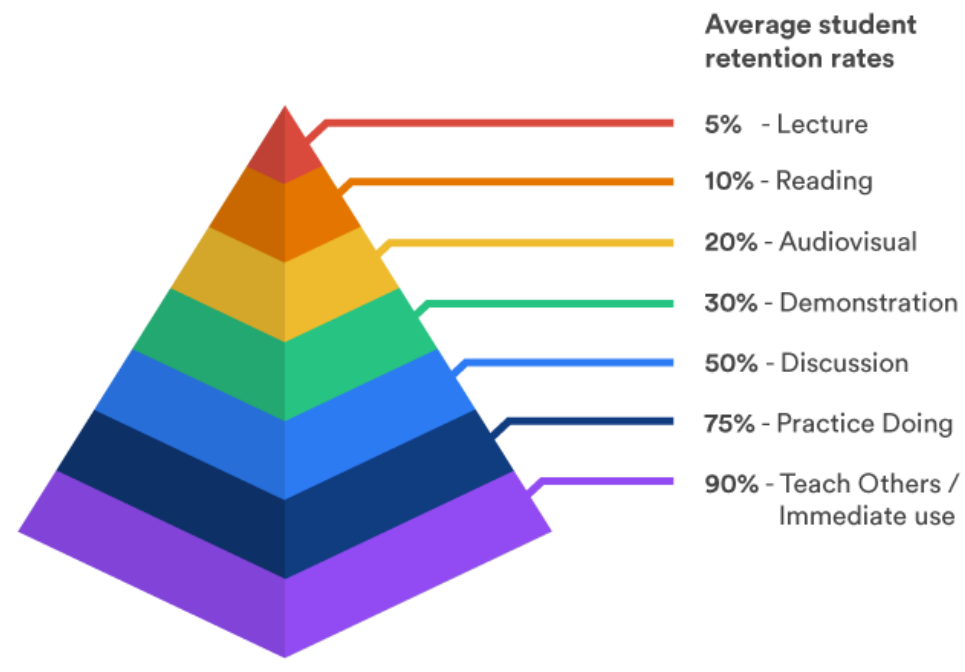

Fuente: Elaboración Propia 


\section{Metodología.}

El experimento inicia proporcionando el material musical de una o varias canciones que incluyen cuatro tipos de trabajo: juego, movimiento, música y visibilidad, en consecuencia, casi todos los que memorizaron las palabras de las canciones preguntaron sobre el significado de las palabras desconocidas Esta metodología justifica que las obras musicales contribuyen al estudio nuevas unidades léxicas y favorecen la comunicación en el idioma inglés. La música se considera uno de los métodos más efectivos de memoria de material linguiístico.

El experimento se llevó a cabo con los 100 estudiantes divididos en tres grupos, el primero un grupo de treinta estudiantes de la Facultad de Administración de Empresas, el segundo, un grupo de 30 estudiantes de la Facultad de Ciencias y el tercer grupo de 40 estudiantes de educación secundaria. El trabajo se llevó a cabo durante 8 semanas casa sesión de trabajo inició con la presentación de una canción distinta que fue escuchada en promedio cinco veces, las canciones más populares escogidas entre los estudiantes fueron las del género pop, también se incluyeron canciones con un contenido amplio de vocabulario. Un ejemplo del tipo de canciones empleado se muestra en la tabla1:

La aplicación del método crea un ambiente de relajación y adquisición inconsciente del conocimiento haciendo que el proceso de aprender inglés sea más interesante para el alumno. Una de las mejores maneras de aprender un idioma es el uso de la música, debido a que escuchar música favorece el desarrollo cognitivo (Tomatis, 1969).

Cuando una persona escucha música varias partes del cerebro funcionan a la vez, y es así como el cerebro se desarrolla activamente, la música es un excelente simulador. La música tiene todas las posibilidades de ser utilizado para formar habilidades fonéticas y léxicas, desarrolla oportunidades creativas en un ambiente agradable. Usando la música se crean ambientes psicológicamente cómodos en el aula.

La entonación y el ritmo aportan en la creatividad de los estudiantes para crear nuevas canciones ampliando el vocabulario y la gramática. Del grupo de estudiantes se escogió una muestra aleatoria de 10 a quienes se les aplicó una evaluación para determinar el dominio del lenguaje inglés. Estos resultados fueron comparados con las calificaciones obtenidas en instrumentos similares las ocho semanas anteriores al experimento.

De esta forma se plantea la hipótesis: Si el método de la musicología contribuyó en el desempeño del estudiante y el desarrollo de las destrezas en los individuos objetos de estudio. Se guardó uniformidad en los instrumentos y tiempos de aplicación en los diferentes grupos a fin de verificar si existe diferencia en los niveles de aprendizaje para grupos de diferente edad. 
Tabla 1 canciones y temas empleados para aprender inglés

\begin{tabular}{|c|c|c|}
\hline No & Tema & Contenido \\
\hline \multirow{21}{*}{1} & \multirow{21}{*}{ El alfabeto } & A is for Actor, B for Ballerina \\
\hline & & $\mathrm{C}$ is for Clown - So let the show begin! \\
\hline & & D is for Daisy, E's for Evergreen, and \\
\hline & & F is for Fuzz on a peaches' skin! \\
\hline & & $\mathrm{G}$ is for Gumballs $\mathrm{H}$ is for Hyena \\
\hline & & I is for Inchworm J's for Jellybeaner! \\
\hline & & Kitten's on the keys, Lion's last in line, \\
\hline & & $\mathrm{M}$ is for my Mom who thinks I've lost my mind! \\
\hline & & CHORUS: \\
\hline & & But that's how I practice my ABC's \\
\hline & & Easily as $1-2-3$ ! \\
\hline & & C'mon let's practice our ABC's \\
\hline & & All you have to do is sing with me: (1st ending) \\
\hline & & $\mathrm{N}$ is for Nacho O's for Onion rings, and \\
\hline & & $\mathrm{P}$ is for Pizza — Let's have lunch! \\
\hline & & Q's Quite Delicious R is for Root Beer \\
\hline & & $\mathrm{S}$ is for my Stomach that's much too stuffed! \\
\hline & & $\mathrm{T}$ is for trumpet U's for ukulele \\
\hline & & V is for voices singing, "Come On Home, Bill Bailey!" \\
\hline & & W's for Whistle, X for xylophone \\
\hline & & Now, let's you and I Zigzag all the way home! \\
\hline \multirow{9}{*}{2} & \multirow{9}{*}{ Animales } & I bought a pretty cat I bought a pretty cat \\
\hline & & My cat pleased me. I fed my cat under that old tree. My cat says 'Fiddle- \\
\hline & & I-fee!' I bought a pretty duck My duck pleased me \\
\hline & & I fed my duck under that old tree. \\
\hline & & My duck says quack, quack. My cat says Fiddle-I-fee! I bought a pretty \\
\hline & & goose. My goose pleased me I fed my goose under that old tree. My goose \\
\hline & & says gaggle, gaggle. My duck says quack, quack. My cat says Fiddle-I-fee! \\
\hline & & I bought a pretty hen. My hen pleased me. I fed my hen under that old tree \\
\hline & & My hen says shimmy shack, shimmy shack. My goose says gaggle, gaggle. \\
\hline \multirow{5}{*}{3} & \multirow{5}{*}{$\begin{array}{l}\text { Partes del } \\
\text { cuerpo }\end{array}$} & This is my favorite pumpkin $(\mathrm{x} 4)$ \\
\hline & & It has one scary nose (one nose) \\
\hline & & Two scary eyes (one, two) \\
\hline & & Three scary teeth (one, two, three) \\
\hline & & Scary, scary, ahh!! \\
\hline
\end{tabular}


This is my favorite pumpkin ( $\mathrm{x} 4$ )

My pumpkin can jump (jump, jump)

my pumpkin can dance (dance, dance)

my pumpkin can spin (spin, spin)

scary, scary, ahhh!!!

This is my favorite pumpkin ( $\mathrm{x} 4$ )

It has one scary nose (one nose)

Two scary eyes (one, two)

Three scary teeth (one, two, three)

This is my favorite pumpkin (x4)

4 seasons in a year

I can name all 4.

Do you wannar' hear?

Let's get ready and say them all.

Winter, spring, summer and fall.

I'm thinking of a season with snowmen and ice.

And if you like sledding,

It's very nice.

4

Estaciones del año

It's very cold.

I need my hat and gloves.

Winter is the season I was thinking of!

I'm thinking of a season where it rains for hours.

Which helps the blooming of the brand new flowers.

It starts to warm up

Which I really love.

Spring is the season I was thinking of!

4 seasons in a year

Christmas Is Coming

Christmas is coming,

The goose is getting fat,

Please put a penny

$5 \quad$ Navidad

In the old man's hat.

If you haven't got a penny,

A ha'penny will do,

If you haven't got a ha'penny,

Then God bless you.

Fuente: Elaboración Propia 


\section{Resultados:}

Tabla 2 resultados obtenidos en la evaluación antes y después del método

\begin{tabular}{ccccc}
\hline Sujeto & $\begin{array}{c}\text { Evaluación } \\
\text { Posterior }\end{array}$ & $\begin{array}{c}\text { Evaluación } \\
\text { antes }\end{array}$ & $\begin{array}{c}\text { Diferencia } \\
\text { D }\end{array}$ & $\begin{array}{c}\text { Diferencia } \\
\boldsymbol{D}^{2}\end{array}$ \\
\hline $\mathbf{1}$ & 10 & 6 & 4 & 16 \\
$\mathbf{2}$ & 18 & 8 & 10 & 100 \\
$\mathbf{3}$ & 16 & 11 & 5 & 25 \\
$\mathbf{4}$ & 22 & 14 & 8 & 64 \\
$\mathbf{5}$ & 14 & 10 & 4 & 16 \\
$\mathbf{6}$ & 25 & 20 & 5 & 25 \\
$\mathbf{7}$ & 17 & 10 & 7 & 49 \\
$\mathbf{8}$ & 22 & 18 & 4 & 16 \\
$\mathbf{9}$ & 12 & 14 & -2 & 4 \\
$\mathbf{1 0}$ & 21 & 13 & 8 & 64 \\
\hline
\end{tabular}

FUENTE: Elaboración propia

De las evaluaciones obtenidas en una escala de calificación de 25 puntos antes y después de la aplicación del método los resultados se muestran en la tabla 2. Donde

$$
N=10 \quad \bar{D}_{o b t}=\frac{\sum D}{N}=\frac{53}{10}=5.3
$$

Hipótesis alternativa: especifica que la música afecta el aprendizaje del idioma inglés. Los puntajes de diferencia de la muestra con la media $\bar{D}_{o b t}=5.3$ forman una muestra aleatoria extraída de una población de diferencias con una media $\mu_{D} \neq 0$

Hipótesis nula: establece que la música non influye en el aprendizaje del idioma inglés. Los puntajes de diferencia de la muestra con una media $\bar{D}_{o b t}=5.3$ forman una muestra aleatoria extraída de una población de diferencias con media $\mu_{D}=0$

Aplicando un nivel de confianza del $95 \% \quad \alpha=0.05$ a dos colas

El diseño es de grupos correlacionados, de manera particular interesa la diferencia que se presenta entre las parejas de datos en vez de los datos sencillos de cada condición. Las diferencias de datos aparecen en la tabla 2. Se ha elegido la prueba del signo o la $t$. con la siguiente información: 


$$
N=10 \quad \bar{D}_{o b t}=5.3 \quad S C_{D}=\sum D^{2}-\frac{\left(\sum D\right)^{2}}{N}
$$

El cálculo de T obtenido $t_{o b t}$ corresponde a:

$$
\begin{gathered}
t_{o b t}=\frac{\bar{D}_{o b t}-\mu}{\sqrt{\frac{S C_{D}}{N(N-1)}}} \\
t_{o b t}=\frac{5.3-0}{\sqrt{\frac{98.1}{10(9)}}} \\
t_{o b t}=\frac{5.3}{\sqrt{1.09}} \\
t_{o b t}=5.08
\end{gathered}
$$$$
S C_{D}=379-\frac{(53)^{2}}{10}
$$$$
S C_{D}=98.1
$$

Si t calculado cae dentro de la región crítica se debería rechazar $H_{0}$ hipótesis nula

$$
\left|t_{o b t}\right| \geq\left|t_{\text {crítico }}\right|
$$

Los grados de libertad corresponde al número de diferencias menos 1 . Así el número de grados de libertad es $N-1=10-1=9, \alpha=0.05$ a dos colas y 9 grados de libertad

$$
t_{\text {crítico }}= \pm 2.262
$$

Como

$$
\left|t_{o b t}\right|>2.262
$$

Rechazamos la hipótesis nula $H_{0}$ y concluimos que la música favorece el aprendizaje del inglés en todos los grupos de prueba.

\section{Conclusiones}

El análisis pedagógico, psicológico y metodológico revelan las siguientes conclusiones:

- Las canciones como uno de los tipos de comunicación verbal son un medio de reposición, asimilación y fortalecimiento vocabulario porque incluye palabras nuevas y expresiones.

- Las canciones se absorben y memorizan significativamente como una construcción gramatical, éstas van siempre acompañadas de diálogos y tareas.

- Las canciones ayudan a mejorar la forma de pronunciación de la lengua extranjera. 


\section{Referencias Bibliográficas.}

Alaminos-Fernández, A. F. (2014). Cuando las letras son música: las canciones en inglés en la publicidad española.

Beltrán, M. (2017). El aprendizaje del idioma inglés como lengua extranjera. Revista Boletín Redipe, 6(4), 91-98.

Bernal Vázquez, J. E. (2010). La Música en la enseñanza-aprendizaje del Inglés.

Bernal, C. (2017). Uso de las TICS y su influencia con la enseñanza-aprendizaje del idioma inglés en los estudiantes del I y II ciclo de la Escuela Académico Profesional de la Facultad de Educación UNMSM-Lima . Doctoral dissertation, UNIVERSIDAD NACI.

Bustos Flores, P. F. (2012). El uso de materiales audiovisuales y su influencia en el aprendizaje del idioma inglés.

Castro Martínez, N. D. (2014). El uso de la Música para la enseñanza del Inglés. El Lipdub.

Darian, S. (1969). Antecedentes de la enseñanza moderna de idiomas: Sweet, Jespersen y Palmer. The Modern Language Journal, 53 (8), 545-550.

Díaz, C. M. (2010). Una fotografía de las cogniciones de un grupo de docentes de inglés de secundaria acerca de la enseñanza y aprendizaje del idioma en establecimientos educacionales públicos de Chile. Folios, 31.

Escobar, E. Y. (2005). La música pop en el desarrollo de las habilidades de escucha y producción oral en inglés. Lenguaje. 33, 159-195.

García-Medall Villanueva, J. A. (2001). La traducción en la enseñanza de lenguas.

Medina, M. M. (2013). La importancia del aprendizaje del idioma inglés a temprana edad. Yachana Revista Científica, 2.

Pérez Morales, J. I. (2008). La evaluación como instrumento de mejora de la calidad del aprendizaje. Propuesta de intervención psicopedagógica para el aprendizaje del idioma inglés.

Sotelo, E. R. (2011). Implementación de una metodología de aprendizaje del idioma inglés basado en la música como recurso didáctico. Bachelor's thesis, Universidad Libre.

Tomatis, A. (. (1969). El oído y el lenguaje . Martínez Roca.

\section{【Liencia}




\section{PARA CITAR EL ARTÍCULO INDEXADO.}

Célleri Quinde, S. P., Logroño Becerra, M. A., Ruiz López, E. R., \& Inca Chunata, E. A. (2020). Estimulación musical para las funciones cognitivas del inglés en la educación $\begin{array}{llll}\text { superior. } & \text { ConcienciaDigital, } & 3(2.1), & 188-198\end{array}$ https://doi.org/10.33262/concienciadigital.v3i2.1.1233

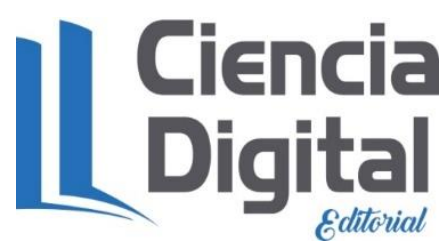

El artículo que se publica es de exclusiva responsabilidad de los autores y no necesariamente reflejan el pensamiento de la Revista Conciencia Digital.

El artículo queda en propiedad de la revista y, por tanto, su publicación parcial y/o total en otro medio tiene que ser autorizado por el director de la Revista Conciencia Digital.

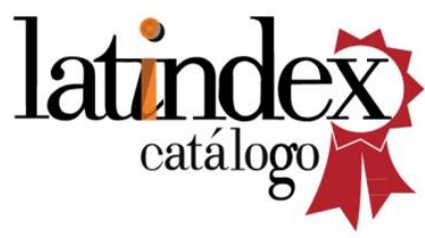

